\title{
Quintessence and the Curvature of the Universe after WMAP
}

\author{
Ralf Aurich and Frank Steiner* \\ Abteilung Theoretische Physik, Universität Ulm, \\ Albert-Einstein-Allee 11, \\ D-89069 Ulm, Germany \\ Received (received date) \\ Revised (revised date)
}

\begin{abstract}
We study quintessence models with a constant (effective) equation of state. It is investigated whether such quintessence models are consistent with a negative spatial curvature of the Universe with respect to the anisotropy of the cosmic microwave background radiation measured by the WMAP mission. If the reionization is negligibly small, it is found that such models with negative curvature are admissible due to a geometrical degeneracy. However, a very high optical depth $\tau$ to the surface of last scattering, as indicated by the polarization measurements of WMAP, would rule out such models.
\end{abstract}

\section{Introduction}

During the last decade, observational cosmology has made enormous progress. In particular, the spectacular discovery in 1992 of the temperature fluctuations of the cosmic microwave background $(\mathrm{CMB})$ radiation by $\mathrm{COBE}{ }^{1}$ has provided important clues about the early Universe and its time evolution. Although the measured anisotropy of the CMB (being of the order of $10^{-5}$ ) is quite small, it sheds important light on several basic physical mechanisms (see Refs. 234 for reviews summarizing the last stand before WMAP). The first findings of NASA's explorer mission "Wilkinson Microwave Anisotropy Probe" (WMAP) [5] has tremendously increased our knowledge of the temperature fluctuations of the CMB, since WMAP has measured the anisotropy of the CMB radiation over the full sky with high accuracy. It is the purpose of this paper to test our theoretical understanding of physical cosmology by making use of the high quality of the WMAP data.

One of the most important results of the cosmological observations made during the last few years has been that all tests of cosmology probing the nature of matter resp. energy in the Universe suggest that our Universe is at the present epoch not dominated by matter but rather by a mysterious energy with negative pressure (see Ref. ${ }^{2}$ for a recent review of the state of the Universe). There are essentially two models to explain this new energy component which nowadays is called dark energy. One possibility is to identify the dark energy with Einstein's cosmological constant

*aurich@physik.uni-ulm.de and frank.steiner@physik.uni-ulm.de 
$\Lambda$ with a corresponding energy density $\varepsilon_{\Lambda}=\Lambda c^{4} /(8 \pi G)$ and negative pressure $p_{\Lambda}=-\varepsilon_{\Lambda}$, assuming a positive cosmological constant. An alternative explanation is quintessence $\frac{678910}{}$, where the dark energy is identified with the energy density $\varepsilon_{\phi}$ arising from a time-evolving scalar (quintessence) field $\phi$ (see Refs. 1112 for recent reviews). An important cosmological parameter is the equation of state of the dark energy component, which in the case of quintessence is given by $w_{\phi}:=p_{\phi} / \varepsilon_{\phi}$, where $p_{\phi}$ denotes the associated pressure. Quintessence models can be considered as generalizations of the cosmological constant and include the latter as the special case $w_{\phi}=$ const. $=-1$. In general, the equation of state $w_{\phi}$ can be a function of redshift. In our following analysis of the WMAP data, we shall assume, however, $w_{\phi}=$ const. $\geq-1$. These models have the property that their quintessence component is negligible at early times such as the recombination epoch. (There are also models with a time-dependent $w_{\phi}$ which lead to "early quintessence" models 13, which are, however, not discussed in this paper.) First of all, a constant equation of state for the dark energy component represents an obvious and also the simplest generalization of the other known energy components of the Universe which have the constant values $w_{\mathrm{r}}=1 / 3$ (for radiation) and $w_{\mathrm{m}}=0$ (for matter). Secondly, as discussed e.g. in Refs. 1415161718, there is an inherent theoretical limitation to determine the time variation of the equation of state of the dark energy component. For example, the luminosity distance $d_{L}$ and the angular-diameter distance $d_{A}$ depend on $w_{\phi}$ through a multiple-integral relation that smears out detailed information about the redshift dependence of $w_{\phi}(z)$ (see Eqs. (51) and (6) below).

One of the most fundamental cosmological parameters is the curvature of the Universe. Many cosmologists seem to accept as established that the Universe is flat corresponding to $k=0$ and $\Omega_{\text {tot }}:=\varepsilon_{\text {tot }} / \varepsilon_{\text {crit }}=1$. Contrary to that opinion, we shall demonstrate that the WMAP data are consistent with certain quintessence models possessing a constant equation of state in a hyperbolic universe, i. e. with negative spatial curvature, $k=-1$, corresponding to $\Omega_{\text {tot }}<1$, provided that the optical depth $\tau$ is negligibly small. Our result is the consequence of the crucial observation that there exists a degeneracy in the space of the relevant cosmological parameters $\left(\Omega_{\mathrm{tot}}, \Omega_{\phi}, w_{\phi}\right)$, where $\Omega_{\phi}:=\varepsilon_{\phi} / \varepsilon_{\text {crit }}$ is the ratio of the quintessence energy density to the critical density.

\section{Quintessence models}

Our background model is the standard cosmological model based on a FriedmannLemaitre universe with Robertson-Walker metric. Then the Friedmann equation reads $\left(a^{\prime}:=d a / d \eta, c=1\right)$

$$
H^{2}:=\left(\frac{a^{\prime}}{a^{2}}\right)^{2}=\frac{8 \pi G}{3} \varepsilon_{\text {tot }}-\frac{k}{a^{2}},
$$

where $a(\eta)$ is the cosmic scale factor as a function of conformal time $\eta$ and $H=H(\eta)$ is the Hubble parameter. The last term in Eq. (11) is the curvature term. Furthermore, $\varepsilon_{\text {tot }}:=\varepsilon_{\mathrm{r}}+\varepsilon_{\mathrm{m}}+\varepsilon_{\phi}$, where $\varepsilon_{\mathrm{r}}$ denotes the energy density of "radiation", i. e. 
of the relativistic components according to photons and three massless neutrinos; $\varepsilon_{\mathrm{m}}=\varepsilon_{\mathrm{b}}+\varepsilon_{\mathrm{cdm}}$ is the energy density of non-relativistic "matter" consisting of baryonic matter, $\varepsilon_{\mathrm{b}}$, and cold dark matter, $\varepsilon_{\mathrm{cdm}}$, and $\varepsilon_{\phi}$ is the energy density of the dark energy due to the quintessence field $\phi$.

In quintessence models, the energy density $\varepsilon_{\phi}(\eta)$ and the pressure $p_{\phi}(\eta)$ of the dark energy are determined by the quintessence potential $V(\phi)$

$$
\varepsilon_{\phi}=\frac{1}{2 a^{2}}{\phi^{\prime}}^{2}+V(\phi) \quad, \quad p_{\phi}=\frac{1}{2 a^{2}}{\phi^{\prime}}^{2}-V(\phi),
$$

or equivalently by the equation of state $w_{\phi}=\frac{p_{\phi}}{\varepsilon_{\phi}}$. The equation of motion of the real, scalar field $\phi(\eta)$ is

$$
\phi^{\prime \prime}+2 \frac{a^{\prime}}{a} \phi^{\prime}+a^{2} \frac{\partial V(\phi)}{\partial \phi}=0
$$

where it is assumed that $\phi$ couples to matter only through gravitation. The various energy densities are constrained by the continuity equation

$$
\varepsilon_{\mathrm{x}}^{\prime}+3\left(1+w_{\mathrm{x}}\right) \frac{a^{\prime}}{a} \varepsilon_{\mathrm{x}}=0
$$

with the constant equation of state $w_{\mathrm{r}}=\frac{1}{3}, w_{\mathrm{m}}=0$ for $\mathrm{x}=\mathrm{r}, \mathrm{m}$, respectively, and $w_{\phi}(\eta)$ for $\mathrm{x}=\phi$. It is worthwhile to remark that the quintessence field $\phi$ may be regarded as a real physical field, or simply as a device for modeling more general cosmic fluids with negative pressure. Since $w_{\phi}$ is assumed to be constant, the potential $V(\phi)$ is uniquely determined as shown in Refs. 1018. However, in solving numerically the coupled Eqs. (1)-(4) no knowledge of the potential $V(\phi)$ is required.

\section{Comparison with the WMAP data}

In our comparison with the WMAP data, we make the following assumptions 2 . The Hubble constant $H_{0}=h \times 100 \mathrm{~km} \mathrm{~s}^{-1} \mathrm{Mpc}^{-1}$ is set to $h=0.70$, and the baryonic density parameter $\Omega_{\mathrm{b}}=0.05$ (i. e. $\Omega_{\mathrm{b}} h^{2}=0.0245$ ) is chosen in agreement with the current Big-Bang nucleosynthesis constraints. Furthermore, the initial curvature perturbation is assumed to be scale-invariant $\left(n_{S}=1\right)$ which is suggested by inflationary models. We have also studied models having a spectral index $n_{S}=0.95$, but the WMAP data alone, i. e. not taking into account large-scale structure data, prefer models having $n_{S}=1$. (We do not consider a $k$-dependent spectral index $n_{S}$ in this paper.) In addition, we have varied the Hubble constant over the range $h=0.64 \ldots 0.72$, but the best models for a fixed curvature all prefer a value close to $h=0.70$. The optical depth $\tau$ to the surface of last scattering is in this Section assumed to be negligibly small. In the next Section, we use the surprisingly high value of $\tau$ found by WMAP 19 . The CMB anisotropy of the quintessence models is computed using the publicly available CAMB code by Antony Lewis and Antony Challinor together with the quintessence module for $w_{\phi}=$ const. models. The angular power spectrum $\delta T_{l}^{2}=l(l+1) C_{l} / 2 \pi$ is compared with the one obtained by 
WMAP 20 which comprises 899 data points. The amplitude of the initial curvature perturbation is determined such that the value of $\chi_{\text {eff }}^{2}$ is minimized. The values of $\chi_{\text {eff }}^{2}$ are obtained by using the FORTRAN code (provided by the WMAP team 21) which, given a theoretical CMB power spectrum, computes the likelihood of that model fit to the WMAP data.
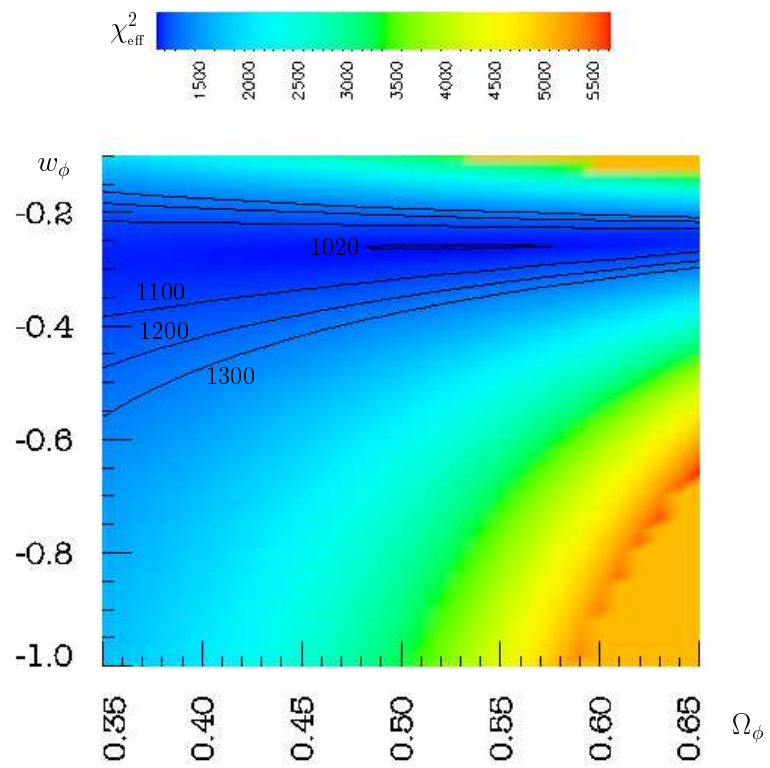

Fig. 1. The $\chi^{2}$ values are shown in dependence on $w_{\phi}$ and $\Omega_{\phi}$ for $\Omega_{\text {tot }}=0.85, h=0.70, \tau=0.0$ and $n_{s}=1.0$ using the WMAP data. The curves with $\chi_{\text {eff }}^{2}=1020,1100,1200$ and 1300 are indicated. The minimum $\chi_{\min }^{2}=1016$ occurs at $\Omega_{\phi}=0.54, w_{\phi}=-0.26$.

Figures 1 to 4 present the values of $\chi_{\text {eff }}^{2}$ for the four cases $\Omega_{\text {tot }}=0.85,0.9,0.95$ and 1.0, respectively, in dependence on $w_{\phi}$ and $\Omega_{\phi}$. Since $\Omega_{\mathrm{tot}}$ is held fixed, the matter density $\Omega_{\mathrm{m}}:=\Omega_{\mathrm{b}}+\Omega_{\mathrm{cdm}}$ is given by $\Omega_{\mathrm{m}}=\Omega_{\mathrm{tot}}-\Omega_{\phi}$ neglecting the small radiation contribution. One observes that a decreasing $\Omega_{\text {tot }}$ demands an increasing $w_{\phi}$, i. e. a less negative value; from $\Omega_{\mathrm{tot}}=0.85$ to $\Omega_{\mathrm{tot}}=1.0$ the equation of state changes from $w_{\phi}=-0.26$ to $w_{\phi}=-1.0$. Among the models considered, the best fit to the WMAP data is obtained for the model with $\Omega_{\mathrm{tot}}=0.95, \Omega_{\phi}=0.61$ and $w_{\phi}=-0.58$ belonging to the minimum at $\chi_{\text {eff }}^{2}=975$, see Figure 3 The corresponding angular power spectrum $\delta T_{l}^{2}$ is shown as a full curve in Figures 5 and 6 and compared with the WMAP measurements. The model with $\Omega_{\text {tot }}=0.90$, $\Omega_{\phi}=0.59$ and $w_{\phi}=-0.36$ is almost of the same quality and belongs to the minimum at $\chi_{\text {eff }}^{2}=985$ (see Figure 2) compared to the 899 data points of WMAP. The corresponding $\delta T_{l}^{2}$ is shown as dashed curve in Figures [5 and [6] Assuming a flat universe, $\Omega_{\mathrm{tot}}=1.0$, one obtains a broad, but extremely flat $\chi_{\mathrm{eff}}^{2}$-valley as shown in Figure 4. The best flat model is obtained for $\Omega_{\phi}=0.68$ and $w_{\phi}=-1.0$ 
Quintessence and the Curvature of the Universe after WMAP 5
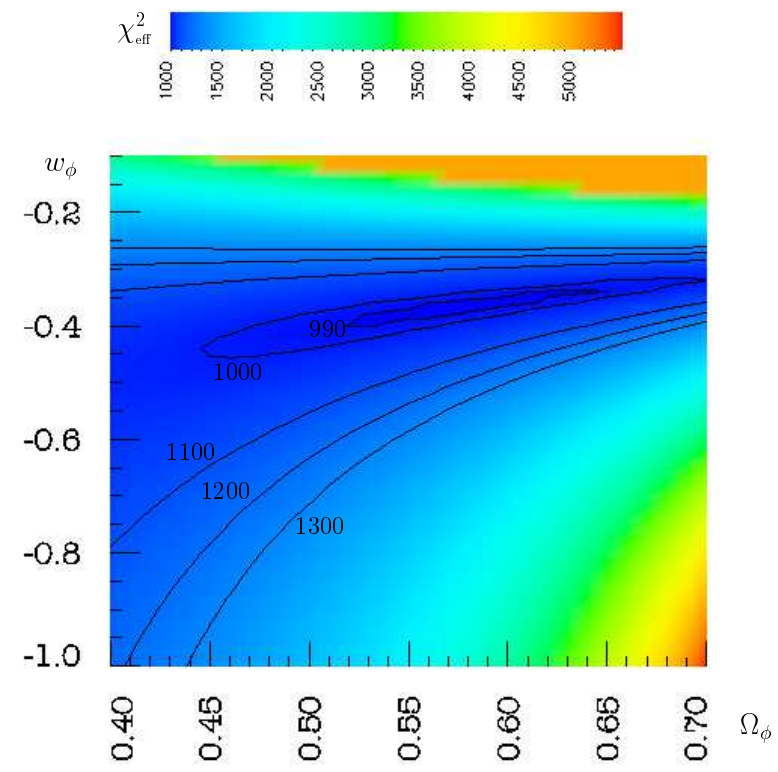

Fig. 2. The same as in Fig.1 for $\Omega_{\text {tot }}=0.9$. The curves with $\chi_{\text {eff }}^{2}=990,1000,1100,1200$ and 1300 are indicated. The minimum $\chi_{\min }^{2}=985$ occurs at $\Omega_{\phi}=0.59, w_{\phi}=-0.36$.

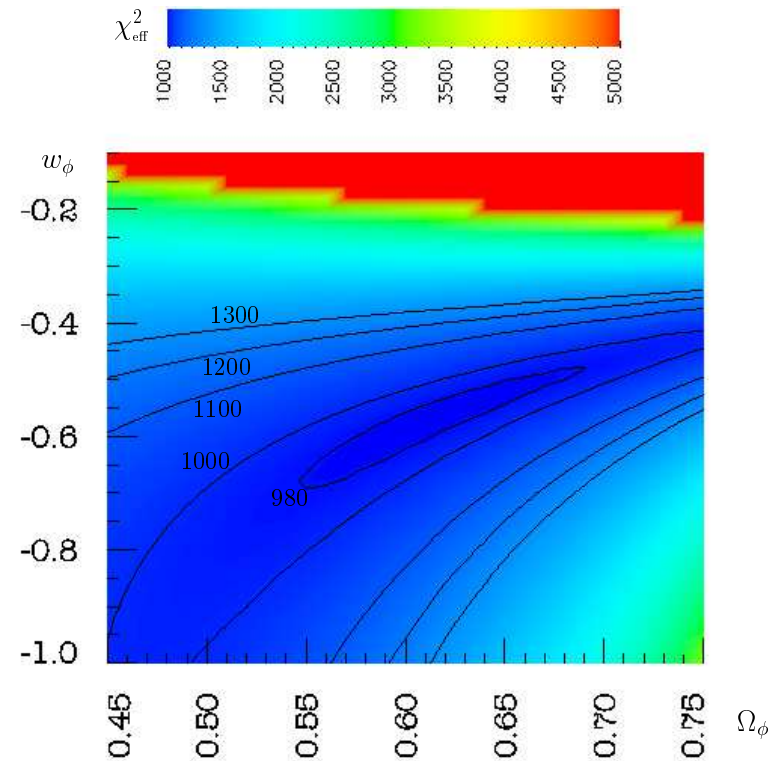

Fig. 3. The same as in Fig. पfor $\Omega_{\text {tot }}=0.95$. The curves with $\chi_{\text {eff }}^{2}=980,1000,1100,1200$ and 1300 are indicated. The minimum $\chi_{\min }^{2}=975$ occurs at $\Omega_{\phi}=0.61, w_{\phi}=-0.58$.

and is plotted as dotted curve in Figures 5 and 6 This model possesses a $\chi_{\text {eff }}^{2}$-value 


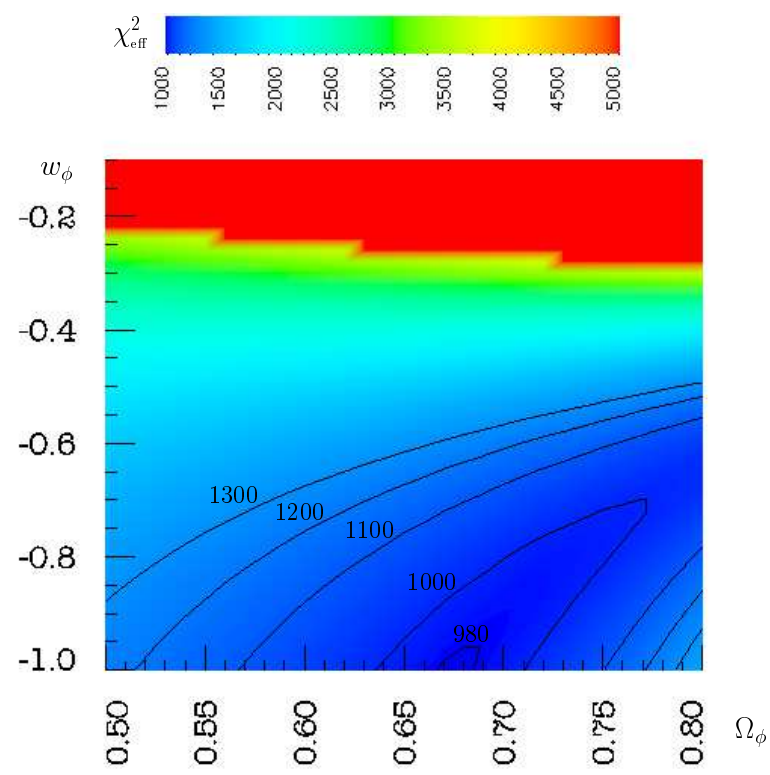

Fig. 4. The same as in Fig.1 for $\Omega_{\text {tot }}=1.0$. The curves with $\chi_{\text {eff }}^{2}=980,1000,1100,1200$ and 1300 are indicated. The minimum $\chi_{\min }^{2}=978$ occurs at $\Omega_{\phi}=0.68, w_{\phi}=-1.0$.

of $\chi_{\text {eff }}^{2}=978$. It is worth noting that the $\chi_{\text {eff }}^{2}$-valleys get increasingly narrower with a horizontal alignment in Figures 14 with decreasing $\Omega_{\text {tot }}$ and thus allow only a small variation in $w_{\phi}$. In the case $\Omega_{\text {tot }}=0.85$, one observes the most confined valley, see Figure 1 where the best model with $\Omega_{\phi}=0.54$ and $w_{\phi}=-0.26$ has $\chi_{\text {eff }}^{2}=1016$. It is seen in Figures 5 and 6 that the best models corresponding to the cases $\Omega_{\text {tot }}=0.85,0.9,0.95$ and 1.0 , respectively, are nearly degenerate with respect to their angular power spectrum $\delta T_{l}^{2}$. The main difference occurs for small values of $l$ where the integrated Sachs-Wolfe effect yields with decreasing $\Omega_{\text {tot }}$ and increasing $w_{\phi}$ a larger contribution. However, this discrepancy scarcely influences the value of $\chi_{\text {eff }}^{2}$ due to the cosmic variance. In contrast, the position of the acoustic peaks and their relative heights are nearly indistinguishable. Even with the high accuracy of the WMAP data, one can hardly discriminate between these models.

The identical position of the peaks is mainly due to a geometrical degeneracy 2218 which arises through the angular-diameter distance $d_{A}$ to the surface of last scattering which has to be computed by $\left(\Omega_{\mathrm{c}}:=1-\Omega_{\mathrm{tot}}\right)$

$$
d_{A}=\frac{1}{H_{0} \sqrt{\left|\Omega_{\mathrm{c}}\right|}\left(1+z_{\mathrm{sls}}\right)} S_{k}\left(H_{0} \sqrt{\left|\Omega_{\mathrm{c}}\right|} \int_{1}^{1+z_{\mathrm{sls}}} \frac{d x}{H(x)}\right),
$$

where $S_{k}(y)$ denotes $\sinh y, y, \sin y$ for $k=-1,0,+1$, respectively. The Hubble parameter $H(x), x=z+1$, is given by

$$
\left(\frac{H(x)}{H_{0}}\right)^{2}=\Omega_{\mathrm{r}} x^{4}+\Omega_{\mathrm{m}} x^{3}-k \Omega_{\mathrm{c}} x^{2}+\Omega_{\phi} e^{3 \int_{1}^{x}\left(1+w_{\phi}\left(x^{\prime}\right)\right) \frac{d x^{\prime}}{x^{\prime}}} .
$$




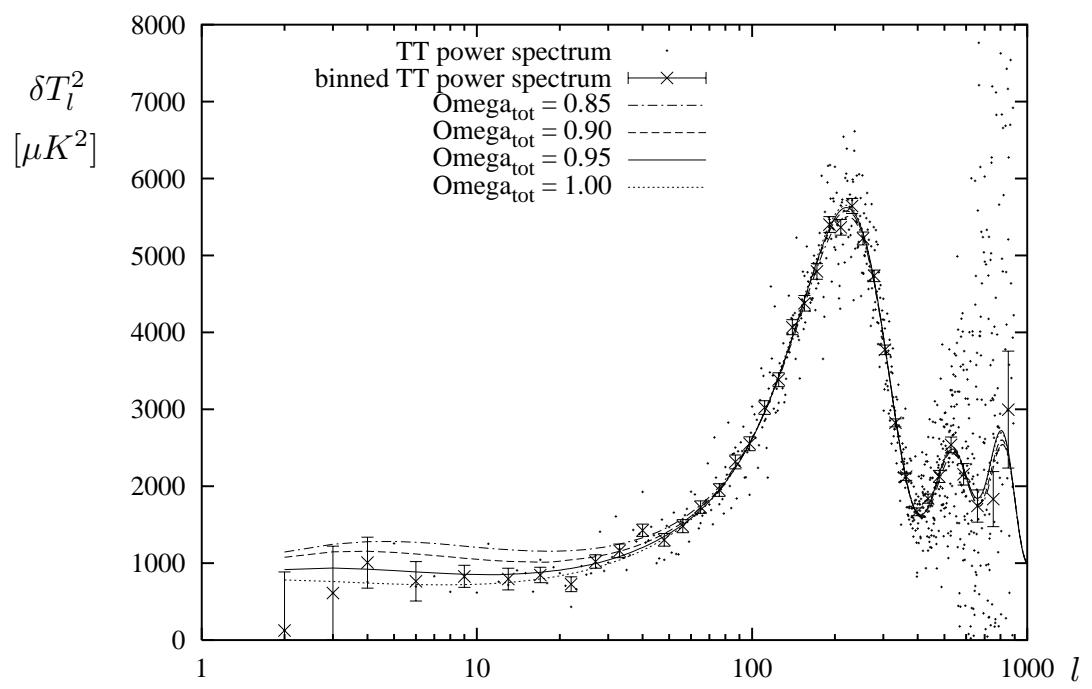

Fig. 5. The angular power spectrum $\delta T_{l}^{2}$ is presented for the four best models for the cases $\Omega_{\mathrm{tot}}=0.85,0.9,0.95$ and $\Omega_{\mathrm{tot}}=1.0$ and compared with the WMAF $[5]$ data. The model fits are determined by the minima of $\chi_{\text {eff }}^{2}$ shown in Figures 1 to 4

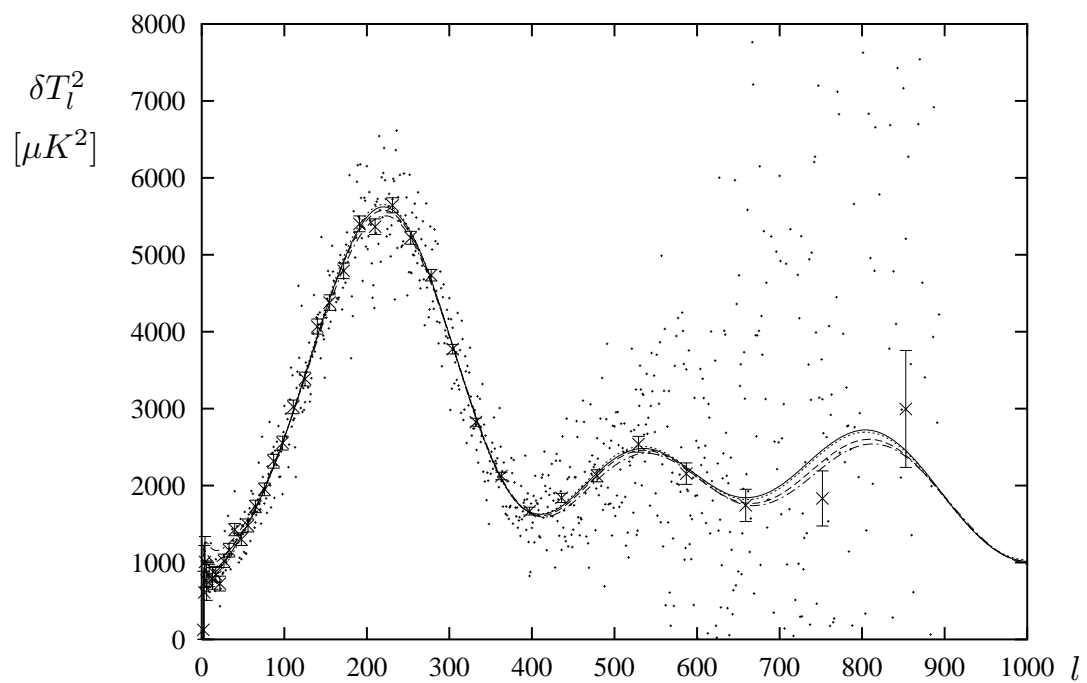

Fig. 6. The same angular power spectra $\delta T_{l}^{2}$ as in Figure 5 are presented in a linear $l$-scale.

The degeneracy due to $d_{A}$ has been discussed by several authors 222316171182425 , in particular in the neighborhood of a flat universe and for $w_{\phi}$ near -1 . One emphasis has been on the dependence of the position $l_{1}$ of the first acoustic peak in the angular power spectrum of the CMB anisotropy on the various cosmological parameters. As a result, it has been concluded that, although there is a degeneracy 


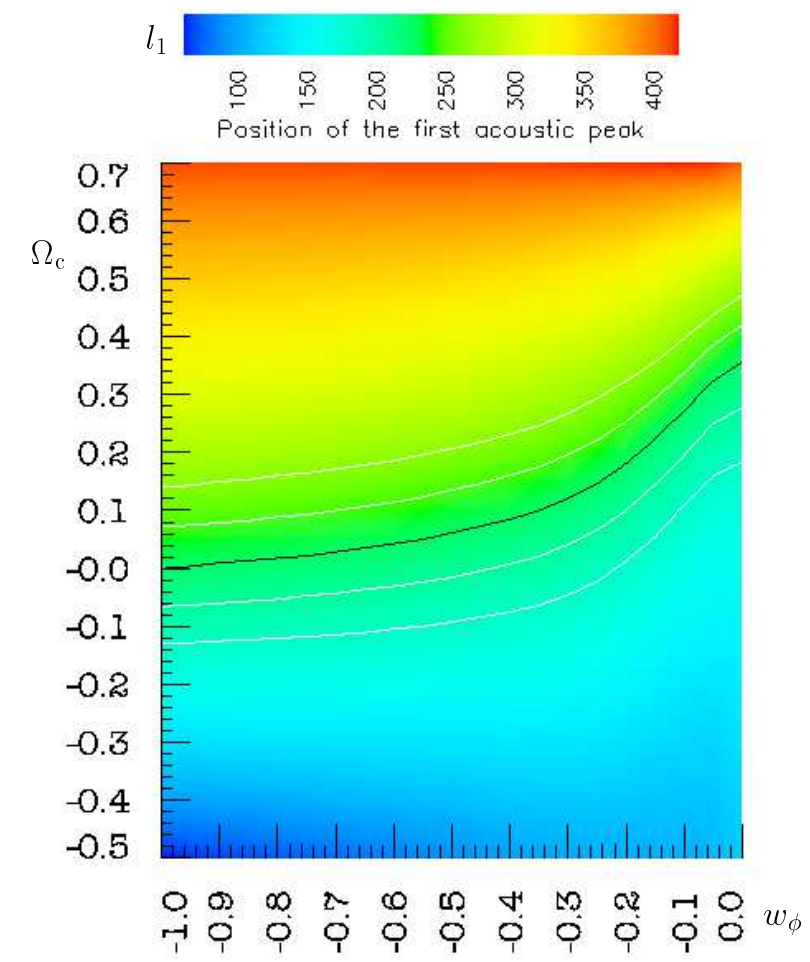

Fig. 7. The position $l_{1}$ of the first acoustic peak is computed in dependence on $w_{\phi}$ and $\Omega_{\mathrm{c}}=$ $1-\Omega_{\mathrm{tot}}$ for $\Omega_{\mathrm{b}}=0.05$ and $\Omega_{\mathrm{cdm}}=0.27$. The white curves indicate the peak position for $l_{1}=180,200,240$ and 260 . The black curve belongs to $l_{1}=220$.

with respect to $\Omega_{\phi}$ and $w_{\phi}$, the position of the first peak is well suited for a determination of the curvature of the Universe. Analyzing the CMB anisotropy and other cosmological observations has led many cosmologists to accept that the Universe is flat in accordance with the inflationary scenarios. However, admitting also quintessence models with $w_{\phi}>-1$ changes this simple picture, since there exists now a degeneracy in the $\left(\Omega_{\mathrm{tot}}, \Omega_{\phi}, w_{\phi}\right)$-space. In Ref. $\frac{18}{18}$ the dependence of $d_{A}$ on the curvature and on $w_{\phi}$ has been investigated using the CMB data before WMAP and it has been shown that there exists a family of models with different curvature having all the same $d_{A}$. In Figure 7 we show the position $l_{1}$ of the first acoustic peak (computed using CAMB) for a large class of models sharing the cosmological parameters $\Omega_{\mathrm{cdm}}=0.27, h=0.70, \tau=0.0$ and $n_{S}=1.0$. The WMAP data confines the position of the first peak close to $l_{1} \simeq 22020$. The models possessing $l_{1}=220$ are connected by the black curve in Figure 7 It is worthwhile to note that the curve for $l_{1}=220$ allows flat models as well as models with negative curvature, but not with positive curvature. Using the WMAP data, the best models, shown in Figures 5 and 6 belong to models lying close to this black curve. The existence of this family in the parameter space $\left(\Omega_{\mathrm{tot}}, \Omega_{\phi}, w_{\phi}\right)$ of equal values of $l_{1}$ is mainly 
due to the degeneracy in $d_{A}$. Under the assumption that the reionization has no significant influence on the CMB anisotropy, we conclude that this geometrical degeneracy allows our Universe to possess negative spatial curvature compatible with the new WMAP data.

\section{Polarization and the influence of early reionization}

WMAP has also obtained data for the correlation of the polarization with the temperature variations 19 . This data requires an unexpected high optical depth $\tau$ to the surface of last scattering of $\tau=0.17 \pm 0.04 \frac{19}{19}$ and $\tau=0.17 \pm 0.0726$. This early reionization erases some of the primary anisotropy below the corresponding horizon (reionization damping) 27 which roughly corresponds to $l \simeq 10$ for the considered models. Thus the angular power spectrum is only slightly altered below $l \simeq 10$ as can be seen in Figure $8 \mathrm{k}$ ) in the case of our two best models for $\Omega_{\text {tot }}=1.0$ and $\Omega_{\text {tot }}=0.9$, respectively. For $l \gtrsim 100$ the reionization damping is almost independent of $l$ and, thus, does not alter the peak structure. The erased power for $l \gtrsim 10$, i. e. the reduced peak height (proportional to $e^{-2 \tau}$ ), is compensated by increasing the initial perturbation amplitude in order to obtain a first peak of the right amplitude. As a consequence the multipoles below $l \simeq 10$ are increased in comparison with models possessing negligible reionization as shown in Figure 8 b). A close inspection of Figure 5 reveals that our models with negative curvature already possess a bit too much power for small values of $l$. These models have been computed with negligible optical depth $\tau=0$ to the surface of last scattering. Thus increasing the optical depth $\tau$ increases the power for small values of $l$ so severely that the models get such high values of $\chi_{\text {eff }}^{2}$ that they are very unlikely.

Before returning to the high optical depth $\tau$, we would like to show in Figure 9 that our models describe the TE-correlation $C_{l}^{\mathrm{TE}}$ of the polarization with the temperature variations for $l \gtrsim 6$ very well. (Note that there are no free parameters,
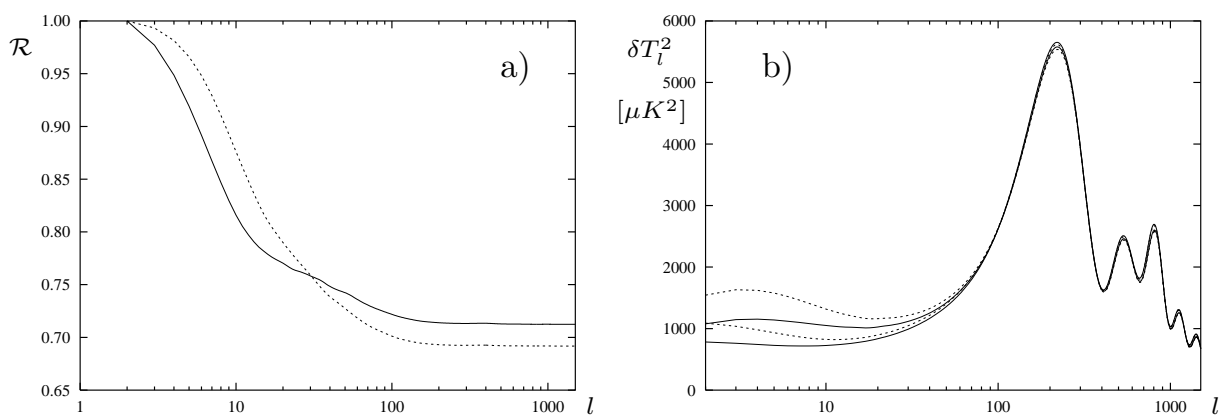

Fig. 8. Panel a) shows the reionization suppression $\mathcal{R}:=C_{l}(\tau=0.15) / C_{l}(\tau=0.0)$ for the two "best" models for $\Omega_{\text {tot }}=1.0$ (solid curve) and $\Omega_{\text {tot }}=0.9$ (dashed curve), if the optical depth $\tau$ is increased from $\tau=0.0$ to $\tau=0.15$. Panel b) shows $\delta T_{l}^{2}$ for these two cases with $\tau=0.0$ (solid curves) and $\tau=0.15$ (dashed curves). The two upper curves at low values of $l$ belong to the case with $\Omega_{\text {tot }}=0.9$. 


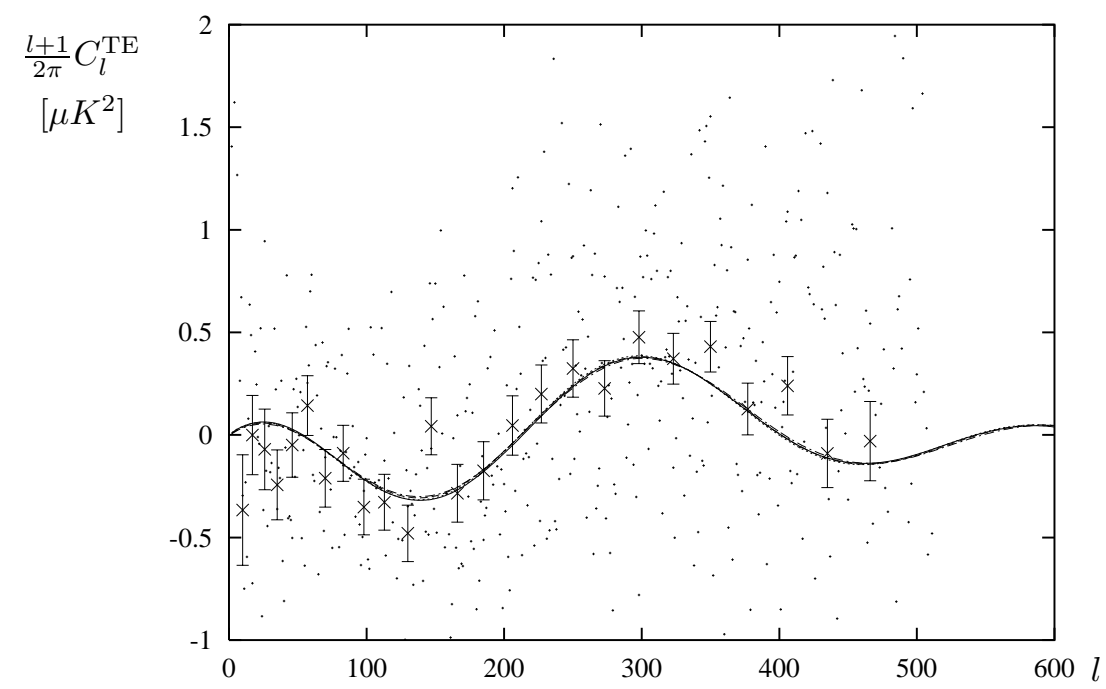

Fig. 9. The cross power spectra $(l+1) C_{l}^{\mathrm{TE}} /(2 \pi)$ are presented for the four best models for the cases $\Omega_{\mathrm{tot}}=0.85,0.9,0.95$ and $\Omega_{\mathrm{tot}}=1.0$ and compared with the WMAF 19 data. The model fits are determined by the minima of $\chi_{\text {eff }}^{2}$ shown in Figures 1 to 4

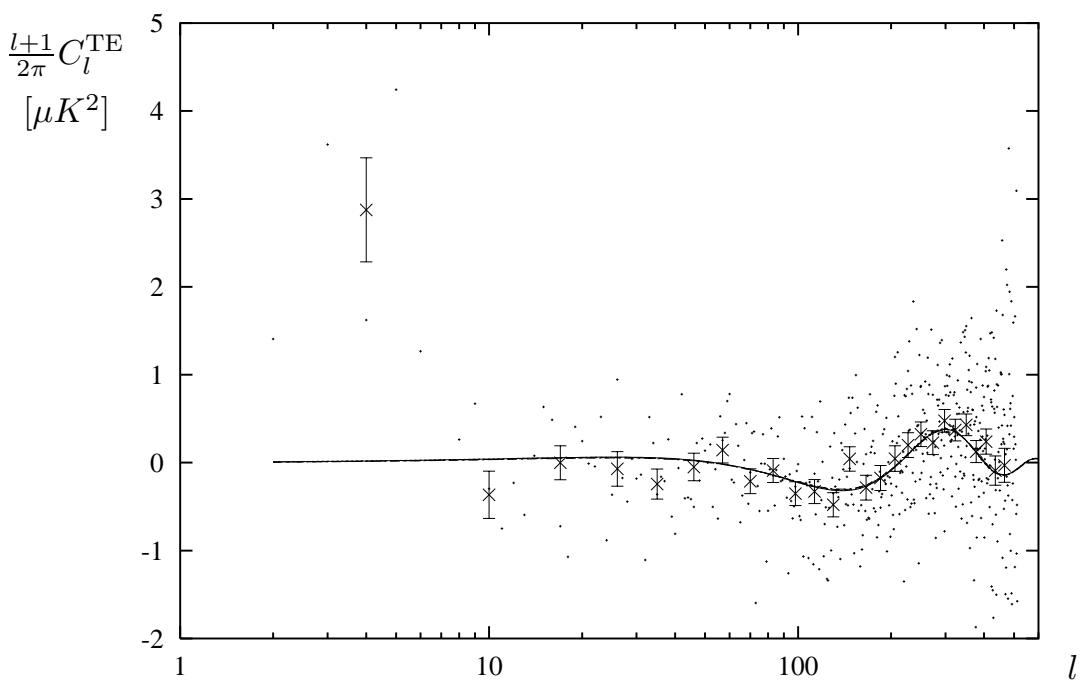

Fig. 10. The same cross power spectra $(l+1) C_{l}^{\mathrm{TE}} /(2 \pi)$ as in Figure 9 are presented in a logarithmic $l$-scale. The large rise of the measured power for $l \lesssim 10$ is an indication for a very early reionization.

since all parameters have already been fixed by the TT power spectrum.) Here the data obtained by WMAP 19 are shown in comparison with the four models discussed above ignoring the reionization. One observes that the oscillations are 
very well described by all four models, and that the models are again degenerated. In order to emphasize the oscillations, we have chosen a scale in Figure 9 which does not show some data points below $l \lesssim 5$. In Figure 10 we show the same data as in Figure 9 using a logarithmic scale. One observes that the models do not describe the high observed values below $l \lesssim 6$. To describe these high values, a large optical depth $\tau$ is required.

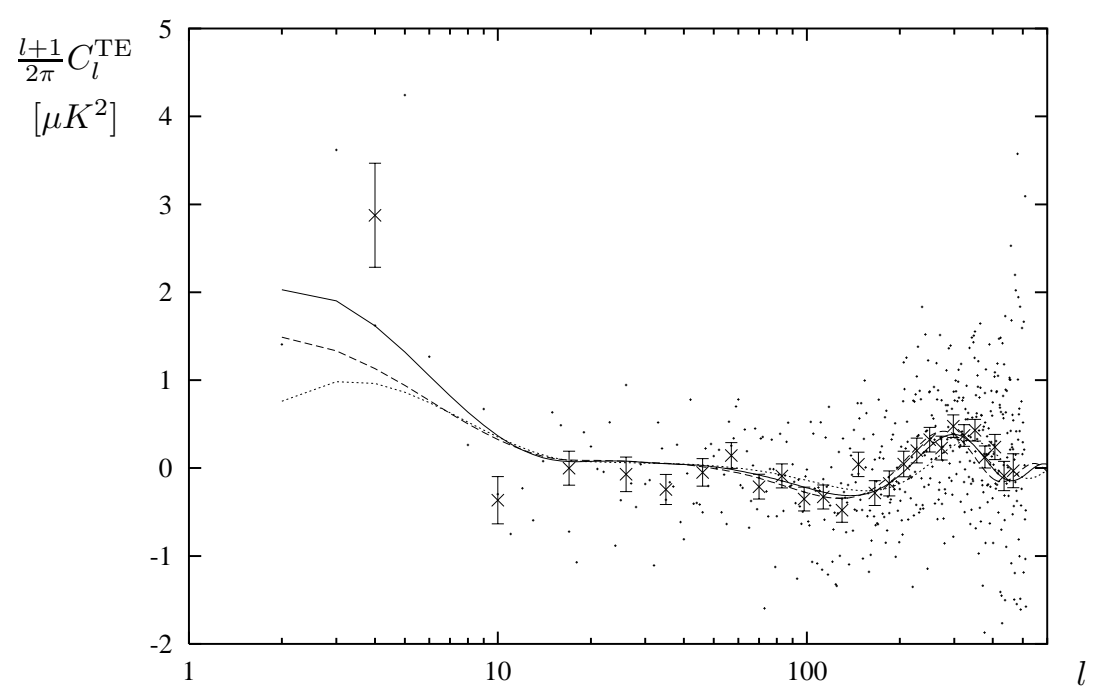

Fig. 11. The cross power spectra $(l+1) C_{l}^{\mathrm{TE}} /(2 \pi)$ are shown for three models with $\tau=0.15$, $\Omega_{\mathrm{b}}=0.05$ and $\Omega_{\mathrm{cdm}}=0.27$. The solid curve belongs to a flat model with $\Omega_{\mathrm{tot}}=1.0, \Omega_{\phi}=0.68$ and $w_{\phi}=-1.0$, the dashed curve to a model which differs from the preceding one only in the increased $w_{\phi}=-0.5$, and the dotted curve belongs to a model where $\Omega_{\phi}$ is reduced to $\Omega_{\phi}=0.58$, such that the model has $\Omega_{\text {tot }}=0.9$.

Figure 11] shows three models with a large optical depth $\tau=0.15$ and with $\Omega_{\mathrm{b}}=0.05$ and $\Omega_{\mathrm{cdm}}=0.27$. The model belonging to the solid curve is a flat model, i. e. having $\Omega_{\phi}=0.68$, with a cosmological constant $w_{\phi}=-1.0$. This model shows the required increase for very small values of $l$. Replacing the cosmological constant by a quintessence component with $w_{\phi}=-0.5$ reduces the power as shown by the dashed curve in Figure 111 Thus quintessence models require an even higher value of $\tau$ in order to obtain the same high level of TE-correlation for low values of $l$. The same behavior is observed if one changes instead of the equation of state $w_{\phi}$ the curvature, i. e. $\Omega_{\mathrm{tot}}$. The dotted curve belongs to a model which differs from the one corresponding to the solid curve by a reduced $\Omega_{\phi}=0.58$, i. e. a model with $\Omega_{\mathrm{tot}}=0.9$. This curve reveals suppressed power at low values of $l$ in comparison to the flat case. Models with negative curvature $\Omega_{\text {tot }}<1$ require also $w_{\phi}>-1$ in order to obtain for the first acoustic peak a position around $l \simeq 220$, as shown in Figure 7 However, as we have just seen, both ingredients, the increased $w_{\phi}$ as 
well as the negative curvature, reduce the level of TE-correlation at low values of $l$. To describe the observed TE-correlation, extremely high values of $\tau$ are required, and thus the reionization damping is in these cases too strong. A Universe with negative curvature would then only be possible if the multipoles at low values of $l$ are exceptional outliers due to the cosmic variance.

The question whether quintessence models with negative curvature are admissible or not thus depends on the optical depth $\tau$. However, there is now some controversy whether a value of the order of $\tau=0.17$ is compatible with physical models of structure formation. In Refs. 2829 it is shown that simple models of reionization are inconsistent with both $\tau=0.17$ and the detection of the Gunn-Peterson trough in high-redshift quasars 3031 . In Ref. 32 a degeneracy between the optical depth $\tau$ and the spectral index $n_{S}$ is used to argue for a lower value of $\tau \simeq 0.11$. In this case a more complicated model involving a time-dependent effective UV-efficiency is shown to be consistent 32 with the WMAP data. In Ref. 33 N-body simulations are presented which match the high value of $\tau$. However, these simulations predict a mass-averaged neutral fraction of $\sim 1 \%$ at $z \simeq 13$ and not at $z \simeq 6$ as required by the high-redshift quasar spectra. As a plausible solution of this problem, an epoch of recombination following the first reionization is suggested 33 in order to have enough neutral matter at $z \simeq 6$. Whether the current observed value of the optical depth withstands future data remains to be seen.

\section{Summary}

In this paper, we have studied quintessence models with a constant (effective) equation of state. Special attention has been paid to the question whether such quintessence models allow negative spatial curvature with respect to the WMAP data. The geometrical degeneracy for this model class is emphasized. Furthermore, it is shown that a very high optical depth $\tau$ to the surface of last scattering would rule out models with negative curvature. If an optical depth around $\tau \simeq 0.17$ will be established, then the Universe can be at most marginally negatively curved. A flat Universe would then be established not by the position of the acoustic peaks, which are equally well described by quintessence models with negative curvature as we have shown, but by the unexpected early reionization. If on the other hand, the optical depth would be established as negligibly small, say around $\tau \simeq 0.05$, then there are quintessence models with negative curvature which can describe the peak structure due to the geometrical degeneracy in the three-dimensional $\left(\Omega_{\mathrm{tot}}, \Omega_{\phi}, w_{\phi}\right)$ space.

\section{Acknowledgements}

We thank Antony Lewis and Antony Challinor for making their CAMB code publicitly available. 


\section{References}

1. G. F. Smoot, C. L. Bennett, A. Kogut, E. L. Wright, J. Aymon, N. W. Boggess, E. S. Cheng, G. de Amici, S. Gulkis, M. G. Hauser, G. Hinshaw, P. D. Jackson, et al., Astrophys. J. Lett. 396, L1 (1992).

2. L. M. Krauss, astro-ph/0301012 (2003), to appear in proceedings, ESO-CERN-ESA Symposium on Astronomy, Cosmology and Fundamental Physics, March 2002.

3. A. Kosowsky, astro-ph/0301131 (2003), to appear in "The Emergence of Cosmic Structure", Proceedings of the 13th Annual Astrophysics Conference in Maryland, eds. S. Holt and C. Reynolds, AIP Press, 2003.

4. X. Wang, M. Tegmark, B. Jain, and M. Zaldarriaga, astro-ph/0212417 (2002).

5. C. L. Bennett, M. Halpern, G. Hinshaw, N. Jarosik, A. Kogut, M. Limon, S. S. Meyer, L. Page, D. N. Spergel, G. S. Tucker, E. Wollack, E. L. Wright, et al., astro-ph/0302207 (2003).

6. M. S. Turner, Phys. Rev. D 28, 1243 (1983).

7. P. J. E. Peebles and B. Ratra, Astrophys. J. Lett. 325, L17 (1988).

8. B. Ratra and P. J. E. Peebles, Phys. Rev. D 37, 3406 (1988).

9. R. R. Caldwell, R. Dave, and P. J. Steinhardt, Phys. Rev. Lett. 80, 1582 (1998).

10. R. Aurich and F. Steiner, Mon. Not. R. Astron. Soc. 334, 735 (2002).

11. V. Sahni and A. Starobinsky, International Journal of Modern Physics D 9, 373 (2000).

12. P. J. E. Peebles and B. Ratra, Rev. Mod. Phys. 75, 559 (2003).

13. R. R. Caldwell, M. Doran, C. M. Müller, G. Schäfer, and C. Wetterich, astroph/0302505 (2003).

14. I. Maor, R. Brustein, and P. J. Steinhardt, Phys. Rev. Lett. 86, 6 (2001), Erratum Phys. Rev. Lett.87(2001) 049901.

15. M. Doran, M. Lilley, J. Schwindt, and C. Wetterich, Astrophys. J. 559, 501 (2001).

16. N. J. Cornish, Phys. Rev. D 63, 027302 (2000).

17. D. Huterer and M. S. Turner, Phys. Rev. D 64, 123527 (2001).

18. R. Aurich and F. Steiner, astro-ph/0212471 (2002), to appear in Phys. Rev. D(15 June 2003).

19. A. Kogut, D. N. Spergel, C. Barnes, C. L. Bennett, M. Halpern, G. Hinshaw, N. Jarosik, M. Limon, S. S. Meyer, L. Page, G. Tucker, E. Wollack, et al., astroph/0302213 (2003).

20. G. Hinshaw, D. N. Spergel, L. Verde, R. S. Hill, S. S. Meyer, C. Barnes, C. L. Bennett, M. Halpern, N. Jarosik, A. Kogut, E. Komatsu, M. Limon, et al., astro-ph/0302217 (2003).

21. L. Verde, H. V. Peiris, D. N. Spergel, M. R. Nolta, C. L. Bennett, M. Halpern, G. Hinshaw, N. Jarosik, A. Kogut, M. Limon, S. S. Meyer, L. Page, et al., astroph/0302218 (2003).

22. G. Efstathiou and J. R. Bond, Mon. Not. R. Astron. Soc. 304, 75 (1999).

23. W. Hu, D. J. Eisenstein, M. Tegmark, and M. White, Phys. Rev. D 59, 23512 (1999).

24. R. Bean and A. Melchiorri, Phys. Rev. D 65, 041302(R) (2002).

25. A. Melchiorri, L. Mersini, C. J. Ödman, and M. Trodden, astro-ph/0211522 (2002).

26. D. N. Spergel, L. Verde, H. V. Peiris, E. Komatsu, M. R. Nolta, C. L. Bennett, M. Halpern, G. Hinshaw, N. Jarosik, A. Kogut, M. Limon, S. S. Meyer, et al., astro$\mathrm{ph} / 0302209$ (2003).

27. W. Hu and M. White, Astrophys. J. 479, 568 (1997).

28. Z. Haiman and G. P. Holder, astro-ph/0302403 (2003).

29. R. Cen, astro-ph/0303236 (2003).

30. R. H. Becker, X. Fan, R. L. White, M. A. Strauss, V. K. Narayanan, R. H. Lupton, 
14 R.Aurich and F.Steiner

J. E. Gunn, J. Annis, N. A. Bahcall, J. Brinkmann, A. J. Connolly, I. Csabai, et al., Astron. J. 122, 2850 (2001).

31. X. Fan, M. A. Strauss, D. P. Schneider, R. H. Becker, R. L. White, Z. Haiman, M. Gregg, L. Pentericci, E. K. Grebe, V. K. Narayanan, Y. Loh, G. T. Richards, et al., astro-ph/0301135 (2003), Astron. J.in press.

32. W. A. Chiu, X. Fan, and J. P. Ostriker, astro-ph/0304234 (2003).

33. B. Ciardi, A. Ferrara, and S. D. M. White, astro-ph/0302451 (2003). 